\title{
Implicaciones familiares y sociales de la muerte materna
}

\author{
Family and social implications of maternal death
}

Leidy Lozano-Avendaño', Alix Zoraida Bohórquez-Ortiz², Gloria Esperanza Zambrano-Plata ${ }^{3}$

1 Enfermera. Integrante Grupo Investigación Salud Pública. Cúcuta, Colombia. e-mail: ladyloav@gmail.com

2 Mg en Salud Pública. Docente catedrática - Directora Grupo de Investigación Salud Pública, Universidad Francisco de Paula Santander. Cúcuta, Colombia e-mail: azbohorq@hotmail.com

3 PhD. en Educación, Magister en Enfermería Materno Perinatal. Docente Facultad Ciencias de la Salud, Integrante Grupo Investigación Salud Pública, Universidad Francisco de Paula Santander. Cúcuta, Colombia. e-mail: gloriae91@hotmail.com

\section{Resumen}

Introducción: La mortalidad materna es considerada un indicador sensible al desarrollo social, porque muchas de estas muertes ocurren por razones evitables, vinculadas a condiciones de pobreza. Hay datos estadísticos sobre el número de muertes maternas a nivel internacional y local, que resaltan la magnitud del problema, así como el conocimiento científico de las causas de estos fallecimientos; pero escasa información publicada sobre el impacto familiar y el desequilibrio que se produce cuando ocurre una muerte materna, situación que trae consigo desconocimiento sobre la real situación de los hijos huérfanos y la familia. Objetivo: Conocer las implicaciones familiares y sociales de la muerte materna a través de la revisión sistemática de la literatura científica publicada. Materiales y métodos: Se realizó una búsqueda en las bases de datos incluidas en los servicios LILACS, ProQuest, MEDLINE y en la biblioteca virtual de salud SciELO. La muestra final fue de 20 artículos. Resultados: Los estudios mostraron que la pérdida inesperada de la madre genera consecuencias emocionales, económicas y de salud en todos los miembros del hogar. Conclusiones: Los hallazgos indican que esta problemática debe ser abordada de manera integral con el fin de mitigar el impacto que genera la muerte materna.

Palabras clave: Muerte materna; huérfano, relaciones familiares; salud mental; niños vulnerables. (Fuente: DeCS, Bireme).

\begin{abstract}
Introduction: Maternal mortality is considered a sensitive indicator of social development, as many of these deaths occur for preventable reasons, linked to poverty. There is statistical data on the number of internationally and locally maternal deaths, which highlights the magnitude of the problem as well as the scientific knowledge of the causes of these deaths; but there is limited published information on the family impact and imbalance that occurs when a maternal death occurs, which leads to lack of knowledge about the real situation of orphaned children and family. Objective: To know the family and social implications of maternal death through a systematic review of the published scientific literature. Materials and methods: A search in the databases including LILACS, PROQUEST, MEDLINE services and virtual health library SCIELO was conducted. The final sample consisted of 20 articles. Results: The studies showed that the unexpected loss of the mother generates emotional, economic and health consequences for
\end{abstract}


all household members. Conclusions: The findings indicate that this problem must be addressed comprehensively in order to mitigate the impact generated by maternal death.

Keywords: Maternal death; orphans; family relations; mental health; vulnerable children; fatherless family. (Source: DeCS, Bireme).

\section{Introducción}

La mortalidad materna es un indicador que refleja las inequidades sociales y económicas de la mujer en la sociedad. Cada día mueren en todo el mundo unas 800 mujeres por complicaciones relacionadas con el embarazo o el parto. En 2013 murieron 289. 000 mujeres durante el embarazo y el parto o después de ellos; prácticamente todas estas muertes se produjeron en países de ingresos bajos y la mayoría de ellas podrían haberse evitado ${ }^{1}$.

La mejora de la salud materna se ha convertido en prioridad para la comunidad internacional, siendo uno de los ocho Objetivos de Desarrollo del Milenio (ODM) donde el gobierno colombiano, junto con 189 países más, se comprometieron en disminuir para 2015, en tres cuartas partes, (75\%) la razón de mortalidad materna (RMM) respecto a la registrada en 1990. Sin embargo, solo se ha disminuido un 2,6\% al año, cifra que está lejos de la reducción del 5,5\% anual para el logro del objetivo ${ }^{2}$.

Según el informe de las Naciones Unidas ${ }^{3}$, los países de América Latina y el Caribe han logrado avances significativos en la reducción de las muertes relacionadas con complicaciones en el embarazo y el parto; lográndola de un $40 \%$ entre 1990 y 2013, poco menos que el promedio mundial (45\%) y por debajo de la meta del $75 \%$ fijada por los ODM y donde Colombia solo ha conseguido un progreso mínimo, con relación al resto de países de América Latina, con una reducción del 17\% de las muertes maternas.

Muchas mujeres mueren de complicaciones que se producen durante el embarazo y el parto o después de ellos. En los países de América predominan las causas obstétricas directas de muerte, que superan el $70 \%$. Entre estas causas médicas primarias figuran la hemorragia (25\%), la septicemia (15\%), las complicaciones del aborto (13\%), la eclampsia (12\%) y el parto obstruido $(8 \%)^{3,4}$. Estas complicaciones están directamente relacionadas con la falta de acceso o utilización de los servicios de maternidad. Además, los servicios a menudo no pueden responder a las situaciones de urgencia y la calidad de su atención es, a veces, deficiente ${ }^{5,6}$.

A la deficiencia en la prestación de servicios de calidad, se suman determinantes de tipo social, económico y cultural, como el bajo nivel de escolaridad, la pobreza, la residencia en zonas rurales, urbanas marginales o de conflicto armado, el desplazamiento forzado; aspectos que reflejan una mayor vulnerabilidad de las mujeres ante las complicaciones obstétricas ${ }^{7,8}$.

La mortalidad materna debe ser vista como una tragedia familiar y social, considerarse más allá de una cifra; las mujeres son irremplazables en una familia, cuando muere, deja a sus hijos huérfanos frente a enormes riesgos que van desde el desamparo, el abandono y el maltrato, hasta poner en riesgo su propia supervivencia; perdiéndose la integridad de la familia ${ }^{9,10}$. Una sociedad privada de la aportación de las mujeres es una sociedad en la que se produciría un deterioro de la vida social y económica, un empobrecimiento de su cultura y una grave reducción de su capacidad potencial de desarrollo ${ }^{11,12}$.

Muchas de las muertes maternas se producen en familias con condiciones de pobreza extrema, hecho que perpetua esta condición, por las consecuencias emocionales, de salud y económicas que ocasiona. La mortalidad materna es un grave problema de salud pública y de violación de derechos humanos ${ }^{13}$.

Ante la necesidad de obtener información acerca de las implicaciones familiares y sociales de la muerte materna, se inicia una revisión de las publicaciones científicas sobre el tema. Esta 
búsqueda permitió reflexionar acerca de la muerte materna, considerando su impacto inmediato y a largo plazo en los miembros de la familia. A partir de esta reflexión, se plantea la necesidad de generar propuestas o políticas de inclusión de estas familias en los procesos saludenfermedad, en espera de un cambio en su estado de salud, con un enfoque importante para los servicios de salud pública y de promoción de la salud y prevención de la enfermedad. El personal de la salud debe conocer la situación por la que atraviesan estas familias, con el fin de desarrollar estrategias y acciones de cuidado encaminadas a las necesidades de los hijos huérfanos, y la familia en general, ofreciendo apoyo en torno a las situaciones que más influyen, evitando así un mayor deterioro en su calidad de vida.

\section{Materiales y métodos}

Se llevó a cabo la revisión de la literatura científica disponible, a través de la consulta de las bases de datos incluidas en los servicios LILACS, ProQuest, MEDLINE y en la biblioteca virtual de salud SciELO, con una ventana de observación desde 1998 al 2013. Para el logro de los objetivos propuestos, inicialmente se utilizaron las palabras claves: "impacto en la salud y familia" (impacts on health and family), "impacto en la salud y medio social" (impacts on health and social environment), no obteniéndose resultados en la búsqueda; finalmente se usaron siguientes descriptores: "Mortalidad materna" (Maternal mortality), "Muerte materna" (Maternal deaht), "Muerte materna y huérfano" (Maternal deaht and orphans), "Muerte materna y familia" (Maternal deaht and family). La búsqueda incluye las publicaciones de investigaciones cualitativas y cuantitativas en revistas indexadas de bases de datos en inglés, portugués y español.

Se ha encontrado muy poca cobertura de la temática, tanto en investigaciones nacionales, en Latinoamérica y el Caribe, como en el resto del mundo. La mayoría de las publicaciones están relacionadas con las causas de la muerte materna, siendo reducidos los estudios que aborden sus implicaciones y/o impactos, por esta razón como estrategia de búsqueda secundaria se revisaron las referencias contenidas en los artículos seleccionados, extrayendo los artículos relevantes que no estaban contenidos dentro de la búsqueda inicial, para su posterior revisión.

La selección de datos de los estudios escogidos se lleva a cabo mediante la elaboración de fichas técnicas en la que se registró la información más importante como: autor, país, objetivo general, diseño metodológico, resultados y conclusiones.

Luego se eliminaron aquellos artículos repetidos. Fueron seleccionados un total de 40 artículos; de estos se escogieron 20 que cumplían con los criterios de muerte materna como tema central y el estudio de las implicaciones familiares y sociales como objeto de estudio. Los resultados obtenidos son presentados en la tabla a continuación, en la cual son identificados autores, títulos de los artículos y el tipo de implicación que abordan.

Tabla 1. Investigaciones sobre el tipo de implicación de la mortalidad materna. 1998-2013

\begin{tabular}{|c|c|c|}
\hline Autor. País, año & Título & Implicaciones \\
\hline $\begin{array}{l}\text { Gomes F, Nakano A, Almeida A, } \\
\text { Matuo. Brasil, } 2006^{14}\end{array}$ & La mortalidad materna en la perspectiva de la familia. & Familiares \\
\hline Sáenz S. Guatemala, $2010^{15}$ & Consecuencias de la muerte materna: estudio de caso en Alta Verapaz & Familiares y sociales \\
\hline $\begin{array}{l}\text { Herrera MC, Cruz JL, Robledo GP, } \\
\text { Montoya G. México, } 2006^{16}\end{array}$ & $\begin{array}{l}\text { La economía del grupo doméstico: determinante de muerte materna entre } \\
\text { mujeres indígenas de Chiapas, México }\end{array}$ & $\begin{array}{l}\text { Familiares } \\
\text { (Económicas) }\end{array}$ \\
\hline $\begin{array}{l}\text { Reyes S, Bobadilla J, Karchmer S, } \\
\text { Martínez L. México, } 1998^{17}\end{array}$ & $\begin{array}{l}\text { Efecto de la muerte materna sobre la dinámica familiar y la sobrevivencia } \\
\text { infantil }\end{array}$ & Familiares y sociales \\
\hline $\begin{array}{l}\text { Zubrick SR, Mitrou F, Lawrence D, } \\
\text { Silburn SR. Australia, } 2011^{18}\end{array}$ & $\begin{array}{l}\text { La muerte materna y las circunstancias psicosociales ulteriores de niños } \\
\text { aborígenes australianos y los jóvenes. }\end{array}$ & Familiares y sociales \\
\hline
\end{tabular}




\begin{tabular}{|c|c|c|}
\hline $\begin{array}{l}\text { Ramírez MF, Rivera M, Durón R, } \\
\text { Aguilar O, et al. Honduras, } 2003^{19}\end{array}$ & $\begin{array}{l}\text { Impacto de la mortalidad materna en la familia, especialmente en la niñez } \\
\text { de Occidente de Honduras. }\end{array}$ & Familiares \\
\hline Portal RE, Perú, $2009^{20}$ & $\begin{array}{l}\text { Consecuencias familiares y sociales de la muerte materna en la sub región } \\
\text { de salud Cajamarca. }\end{array}$ & Familiares y sociales \\
\hline Case A, Ardington C. África, $2006^{21}$ & $\begin{array}{l}\text { El impacto de la muerte de los padres sobre los resultados escolares: } \\
\text { evidencia longitudinal de Sudáfrica. }\end{array}$ & Familiares \\
\hline $\begin{array}{l}\text { Wang H, Ye F, Wang Y, Huntington } \\
\text { D. Chna, } 2013^{22}\end{array}$ & $\begin{array}{l}\text { Impacto económico de la mortalidad materna en los hogares en China } \\
\text { rural: un estudio de cohorte prospectivo. }\end{array}$ & $\begin{array}{l}\text { Familiares } \\
\text { (Económico) }\end{array}$ \\
\hline $\begin{array}{l}\text { Ye F, Wang H, Huntington D, Zhou H, } \\
\text { Li Y, You F, Li J, Cui W, Yao M, Wang } \\
\text { Y. China, } 2012^{23}\end{array}$ & $\begin{array}{l}\text { El impacto económico inmediato de las muertes maternas en los hogares } \\
\text { chinos rurales }\end{array}$ & Familiares \\
\hline $\begin{array}{l}\text { Chen SH, Chen Y, Liu J. Estados } \\
\text { Undos, } 2009^{24}\end{array}$ & $\begin{array}{l}\text { El impacto de la inesperada muerte materna en Educación: Primera } \\
\text { Prueba de Tres datos administrativos Enlaces Nacionales }\end{array}$ & Familiares y sociales \\
\hline Pelloso S, Guarino M. Brasl, $2006^{25}$ & La muerte materna una problemática familiar & Familiares \\
\hline $\begin{array}{l}\text { Pavard S, Gagnon A, Desjardins B, } \\
\text { Heyer E. Canada, } 2005^{26}\end{array}$ & $\begin{array}{l}\text { Muerte de la madre y supervivencia infantil: El caso de principios de } \\
\text { Québec. }\end{array}$ & Familiares y sociales \\
\hline Atrash HK. Brasil, $2011^{27}$ & La muerte de los padres y sus implicaciones para la supervivencia infantil & Familiares y sociales \\
\hline $\begin{array}{l}\text { Clark SJ, Kahn K, Houle B, Arteche A, } \\
\text { Collinson MA, Tollman SM. Africa, } \\
2013^{28}\end{array}$ & $\begin{array}{l}\text { Probabilidad de los niños pequeños de morir antes y después de la muerte } \\
\text { de su madre: un estudio de vigilancia de base poblacional del sur rural } \\
\text { africano. }\end{array}$ & Familiares y sociales \\
\hline $\begin{array}{l}\text { Mishra V, Arnold F, Otieno F, Cross } \\
\text { A, Hong R, Kenia, } 2007^{29}\end{array}$ & $\begin{array}{l}\text { Estado de la educación y la nutrición de los huérfanos y los niños de padres } \\
\text { infectados por el VIH en Kenia }\end{array}$ & Familiares \\
\hline $\begin{array}{l}\text { Ronsmans C, Chowdhury ME, } \\
\text { Dasgupta SK, Ahmed A, Koblinsky M } \\
\text { Bangladesh (Asia), } 2010^{30}\end{array}$ & $\begin{array}{l}\text { Efecto de la muerte de los padres en la supervivencia infantil en } \\
\text { Bangladesh rural: un estudio de cohorte }\end{array}$ & Familiares y sociales \\
\hline $\begin{array}{l}\text { Karam M, Bustamante P, } \\
\text { Campuzano M, Camarena A et al. } \\
\text { México, 200731 }\end{array}$ & $\begin{array}{l}\text { Aspectos sociales de la mortalidad materna. Estudio de caso en el Estado } \\
\text { de México }\end{array}$ & Sociales \\
\hline $\begin{array}{l}\text { Yusuf HR, Atrash HK, Estados } \\
\text { Unidos, } 2010^{32}\end{array}$ & La muerte de los padres y la supervivencia de sus hijos. & Familiares \\
\hline Elegbeleye AO. Nigeria, $2013^{33}$ & $\begin{array}{l}\text { Los predictores de la salud mental de los huérfanos y niños vulnerables en } \\
\text { Nigeria. }\end{array}$ & Sociales \\
\hline
\end{tabular}

\section{Resultados y discusión}

La Organización Mundial de la Salud define a la muerte materna como "la muerte de una mujer mientras está embarazada o dentro de los 42 días siguientes a la terminación del embarazo, independientemente de la duración y lugar del mismo, debida a cualquier causa directamente relacionada o agravada por el embarazo o por su manejo"; y a la muerte materna tardía, como "la muerte de una mujer por causas obstétricas directas o indirectas después de los 42 días pero antes de un año de la terminación del embarazo $^{34,35}$.

Considerada como un evento de interés en salud, porque genera desequilibrios sociales y del sistema familiar, lo que hace que la funcionalidad de la familia sea más vulnerable a la aparición de problemas emocionales y de salud.

La muerte materna es el resultado de un sistema de salud poco accesible y culturalmente inadecuado. Es la manifestación de una larga 
cadena de penurias y de factores como: poca escolaridad de las niñas, desnutrición, matrimonio precoz, falta de información, limitado acceso a métodos anticonceptivos y a la condición y situación de subordinación de las mujeres. Todos estos factores, en forma individual y colectiva, contribuyen a la vulnerable situación de las mujeres antes y durante el embarazo, parto y el postparto, incrementando los riesgos y complicaciones propias de la reproducción ${ }^{36}$.

\section{Implicaciones familiares}

La ausencia repentina y permanente de la mujer en el hogar es un factor en la ruptura familiar, los niños a menudo son entregados entre los miembros de la familia; siendo los abuelos los que generalmente asumen su cuidado, estando en particular desventaja, dado su nivel de pobreza y sus escasas posibilidades de generar ingresos, situación que perpetúa riesgos ${ }^{14,18,37}$.

Los hallazgos de Ramírez ${ }^{19}$, demuestran que posterior al evento hubo fragmentación familiar, donde las familias afectadas emigraron a otros hogares y unas familias adoptaron completamente a otras. En la mayoría de los estudios los huérfanos se quedaron con su padre, abuelos, o se distribuyen entre parientes; sin embargo algunos de los hijos viven con otras personas que no tienen una relación de parentesco directo con alguno de los padres, o en total abandono, viviendo en condiciones de miseria, dependiendo de la comunidad para su supervivencia ${ }^{20,38}$.

La alteración en la dinámica familiar origina cambio en los roles, responsabilidades y actividades domésticas, las hijas jóvenes muchas veces deben asumir el cuidado de los huérfanos menores, dejando de lado la escuela15,16,21, convirtiéndose en la madre sustituta. Lo anterior, evidenciado en el estudio realizado por Reyes ${ }^{17}$, donde los hijos huérfanos tuvieron que cambiar las actividades que realizaban previamente, dejar de asistir a la escuela, empezar a trabajar y cuidar a sus hermanos, desarrollando roles para los cuales no estaban preparados.

La mujer es el eje del hogar y en muchos hogares puede ser el único o más importante sostén familiar; su muerte representa una pérdida económica para la familia por ser un miembro activo en la producción ${ }^{14}$; genera un impacto adverso en la economía de los hogares, reflejado en la disminución de los ingresos, aumento de gastos, deudas más altas, y un índice de riqueza más bajo ${ }^{22,23}$.

Debido a que una gran proporción de mujeres en edad fértil pertenece a la población trabajadora y económicamente activa, las muertes maternas generan una pérdida de la productividad económica y una disminución en los ingresos familiares. Si tomamos en cuenta que en países como el nuestro hay una gran proporción de mujeres cabeza de familia que llevan las riendas de sus núcleos familiares y soportan la economía del hogar, la muerte de éstas genera en sus hijos no sólo una carencia de los recursos básicos para la supervivencia sino también carencias afectivas y de protección ${ }^{39}$.

Es evidente que la alta carga económica que causa la muerte de la madre se traduce en una crisis económica grave en el corto y largo plazo, perpetuando la pobreza. $\mathrm{Ye}^{23}$, en su estudio encontró que los costos directos totales con la muerte materna fueron diez veces mayores en comparación con los hogares con parto sin pérdida materna.

Con la muerte materna, los miembros del hogar deben adoptar nuevos roles y responsabilidades, quien se encargue del cuidado de los huérfanos tendrá que abandonar sus actividades productivas, con la consecuente reducción de ingresos, limitando el acceso a la educación, a una buena alimentación y a servicios médicos asistenciales ${ }^{40}$.

Además, del impacto económico y de la fragmentación familiar, este evento afecta emocionalmente a la familia, generando sentimientos de dolor, rechazo y angustia. Si la madre fallece en un hogar, aparte de la tristeza, una de las consecuencias emocionales más claras es la soledad en la que quedan los huérfanos ${ }^{15}$. Entre las reacciones inmediatas predominan los síntomas de ansiedad (inquietud, miedos, fobias y alteraciones del sueño) y las alteraciones del humor (irritabilidad y tristeza) que parecen 
afectar por igual a niños y niñas de todas las edades, aunque su expresión varíe de acuerdo al desarrollo cognitivo y emocional ${ }^{14,41}$. En momentos posteriores, se aprecian alteraciones del comportamiento (inquietud, agresividad y dificultad en la aceptación de normas) junto a problemas de rendimiento y adaptación escolar17,41. Los huérfanos tienen menos probabilidades de matricularse en la escuela y completar su escolaridad 24,38. Es además, una experiencia traumática que puede tener ramificaciones potenciales para el desarrollo y el bienestar del niño, en especial cuando no se proporcionan altos niveles de apoyo ${ }^{18}$.

Se ha encontrado mayor riesgo de problemas de drogadicción, alcoholismo, delincuencia e intentos de suicidio en niños y jóvenes cuya madre biológica ha muerto ${ }^{17,38}$, reflejando la gravedad de este evento, que impacta negativamente a los huérfanos y trae repercusiones en su vida a lo largo del tiempo, siendo éstas mayores, si no cuentan con una red de apoyo o se encuentran en total abandono.

Así mismo, los viudos reflejan sentimientos de dolor, desesperación y miedo al futuro ${ }^{14,41}$, sentimientos que pueden permanecer en el tiempo. El dolor de la pérdida viene cargado de sentimientos de confusión y de la constatación de la desigualdad y el abandono de la atención ${ }^{25}$. Herrera ${ }^{16}$, considera que las implicaciones no terminan con las consecuencias inmediatas del deceso y de los factores que contribuyeron a él, sino que se extiende hasta las complejas secuelas emocionales de la pérdida de la hija, la esposa y la madre, y persiste en el curso del tiempo con efectos tan devastadores como la muerte del recién nacido y un futuro incierto para los hijos huérfanos.

El aumento de la mortalidad de los huérfanos de madre sigue siendo significativo durante la infancia y los primeros años de la adolescencia, que se explica principalmente por la pérdida de la atención materna y por la degradación de las condiciones del hogar, conduciendo a una falta de atención médica, que aumenta la mortalidad en los niños 37,26 .
La orfandad es una de las consecuencias más implacables que expone a grandes riesgos la vida, el desarrollo y el crecimiento de un niño ${ }^{15,20}$. Se ha encontrado que la mortalidad materna e infantil, están altamente correlacionadas; la muerte materna se asocia con 2 a 50 veces mayor riesgo de muerte entre los menores de 5 años 26,27 ; este riesgo aumenta con la presencia de infección por $\mathrm{VIH}^{28,42}$. Nakiyingi encontró que en el año previo y posterior a la muerte de la madre, los niños experimentan tasas de mortalidad cinco veces más altas que aquellos con madres que viven ${ }^{42}$.

Los efectos devastadores de la muerte de la madre en la supervivencia de los niños puede ser probablemente por la interrupción brusca de la lactancia materna, pero la persistencia de los efectos de hasta 10 años de edad, sugieren que la falta de atención materna podría ser un factor crucial $29,30,43$. Muchas de las muertes infantiles cuyas madres habían fallecido estaban asociadas con la desnutrición, lo que sugiere que el papel principal de la madre como fuente de nutrición no fue compensado ${ }^{39,29}$.

Becher ${ }^{44}$ confirma la importancia de los principales grupos de riesgo para la mortalidad infantil: el nivel socioeconómico, las condiciones de salud ambiental, el estado nutricional, la alimentación infantil y la utilización de los servicios de salud; todos esos factores se agravan cuando ocurre una muerte materna; lo que conlleva al incremento en la morbi-mortalidad infantil ${ }^{41}$.

La pérdida de un padre por muerte o deserción es un aspecto importante de la vulnerabilidad, si se suman otros factores tales como, pobreza, hambre, la falta de acceso a los servicios, ropa inadecuada o refugios, hacinamiento, cuidadores deficientes ${ }^{20,45}$; las consecuencias en la salud física, mental y social de los huérfanos serán mayores. Por estas razones la muerte materna, además de ser un problema en salud pública, es un imperativo ético por resolver ${ }^{32,46 .}$

\section{Implicaciones sociales}

La muerte materna está asociada a precarias condiciones sociales, ocurriendo generalmente en familias marginadas, donde hay limitada 
disponibilidad y calidad de servicios de salud, situación que ocasiona un mayor deterioro familiar y social ${ }^{36,47}$.

Con la fusión de la nueva familia se condiciona a un mayor hacinamiento, mayor propensión a enfermedades, mayor riesgo de muerte, y desnutrición ${ }^{38}$. Los huérfanos y especialmente los recién nacidos que quedan vivos son los que sufren las consecuencias directas de este evento, porque no solo pierden su madre, sino que en el país no hay políticas claras de protección para estos huérfanos, de modo que no hay como asegurarles la leche maternizada, la permanencia en sus régimen de afiliación cuando era la madre la cotizante, ni el acompañamiento por las áreas de salud mental 48 .

Las consecuencias de la mortalidad materna son graves para la familia y la comunidad, por lo general, el neonato también fallece en el curso del primer año de vida, convirtiéndose en otro problema de salud pública; las niñas y niños pequeños no reciben los cuidados necesarios para su óptimo desarrollo, y la sociedad se ve privada de un recurso humano cuya labor remunerada y no remunerada es fundamental para el desarrollo humano ${ }^{49}$.

El carecer de acceso a la educación y otros servicios, así como crecer en los márgenes de la sociedad en condiciones de pobreza extrema; ocasiona que los huérfanos puedan difícilmente convertirse en miembros productivos de la sociedad como adultos ${ }^{50}$.

La pobreza y el desempleo favorecen el incremento de "niños de la calle", que a su vez incrementa el riesgo de problemas de drogadicción, alcoholismo y vandalismo ${ }^{31,38}$. Dado que el hijo mayor ante la pérdida de la madre, tiene que asumir muchas veces la responsabilidad de cuidar a sus hermanos pequeños, dejando sus estudios por trabajar, situación que contribuye al incremento de los índices de analfabetismo, que a futuro se verá reflejado en menores oportunidades, menor productividad, acentuando las condiciones de pobreza. Además de todo esto, a las hijas huérfanas, se les prepara de forma indirecta para que en el futuro tengan pareja más tempranamente ${ }^{19}$, contribuyendo al embarazo a temprana edad, que unido a otros factores, crea un círculo vicioso para la muerte materna.

Por todo ello, la muerte materna ha sido reconocida como un problema social en la medida que su ocurrencia está ampliamente relacionada con pobreza y marginación, perpetuando esta condición en las familias donde ocurre.

\section{Conclusiones}

La investigación de la muerte materna y sus implicaciones sociales $y$ familiares aún se encuentra en desarrollo. Con frecuencia los estudios, se centran en conocer las causas de la muerte, para orientar acciones hacia la prevención, sin embargo, cuando ya ocurre debe orientarse el actuar hacia el reconocimiento de la familia donde ha ocurrido una muerte materna, como población vulnerable, con una problemática que afecta seriamente a los hijos huérfanos.

Muchas instituciones a nivel internacional como la OMS, la UNICEF y el Banco Mundial reconocen la magnitud de una muerte materna, no solo por la vida que se pierde, sino también porque este evento afecta a la familia y sociedad, en especial a los huérfanos que quedan desprotegidos, más predispuestos a no recibir atención médica oportuna, y educación a medida que crecen.

El resultado evidente de la muerte de una mujer es la pérdida de su contribución a su familia y su comunidad: el mantenimiento de la familia, la protección de la salud y facilitar la educación de los niños, y la obtención de ingresos.

La muerte materna es un evento trágico e inesperado para todos los integrantes de la familia; no obstante; en los estudios revisados se concluye que son los hijos huérfanos los que sufren las mayores consecuencias, mayor riesgo de morir, de enfermarse, menos probabilidades de recibir atención médica y de asistir a la escuela, implicaciones emocionales que pueden repercutir por mucho tiempo. 
El conocer las implicaciones de la muerte materna, contribuye a la creación de políticas integrales de salud y bienestar, con la participación activa de los diferentes actores y sectores de la sociedad; que aporten al mejoramiento de la calidad de vida de las familias y de la población, haciéndola beneficiaria de la actividad académica e investigativa, que conduzcan a materializar el propósito final de mejorar las condiciones de salud y bienestar de la infancia y la familia, y por ende de la sociedad en general.

Conflicto de intereses: Ninguno declarado por autores.

\section{Referencias}

1. Organización Mundial de la Salud. Mortalidad materna. Centro de prensa. Nota descriptiva $\mathrm{N}^{\circ} 348$ Mayo de 2014 Centro de prensa de la OMS. Disponible en: http://www.who.int/mediacentre/factsheets/fs348/es

2. Organización Mundial de la Salud. ODM 5: Mejorar la salud materna [En línea]. 2012 [aprox. 2 pantallas]. Disponible

en: http://www.who.int/topics/millennium_development_ goals/maternal_health/es/index.html

3. Organización Panamericana de la Salud. Mortalidad materna y neonatal en ALC y estrategias de reducción. [En línea]. 2012. [aprox. 19 pantallas]. Disponible en: http://www.who.int/pmnch/activities/sintesis_situaci onmortalidad_en_alc.pdf

4. Universidad Nacional de Colombia. Boletín del Observatorio en Salud: Sobre la mortalidad materna en Colombia. [En línea]. 2012;5(2):1-7. Disponible en: http://www.revistas.unal.edu.co/index.php/bos/articl e/viewFile/29555/29775

5. Tavara OL. Tendencia de la mortalidad materna en el Perú: desafíos pendientes. Rev. Perú. Ginecol. Obstet. 2013;59(3):157-160.

6. Palacio M. El estancamiento de la mortalidad materna: un problema evitable: Colombia. Bogotá: Organización Panamericana de la Salud. 2004.

7. República de Colombia. Ministerio de la Protección Social. Plan de choque para la reducción de la mortalidad materna [En línea]. 2010. [aprox. 28 pantallas]. Disponible http://www.ossyr.org.ar/pdf/bibliografia/5.2.pdf

8. Marín BA, Dussán LC, González LJ, Benjumea RM, Arengas CI. Mortalidad materna en el Departamento de Caldas (Colombia), durante el quinquenio 2004-2008. Hacia la Promoción de la Salud. 2010;15(1):110-126

9. Carrillo A. Mortalidad materna en Colombia: Reflexiones en torno a la situación y la política pública durante la última década. Rev. Cienc. Salud. 2007; 5(2):72-85.

10. Rivera J, González Inés. Mortalidad Materna: Un problema de salud pública y derechos humanos [En línea]. 2003. [aprox. 260 pantallas]. Disponible en: http://www.unicef.org/argentina/spanish/ar_insumos _mortalidadmaterna.pdf

11. Anderson FWJ, Morton SU, Naik S, Gebrian B. Maternal mortality and the consequences on infant and child survival in rural Haiti. Matern Child Health J. 2007; 11(4):395-401.

12. Organización Panamericana de la Salud. Reducción de la mortalidad y la morbilidad maternas: Consenso Estratégico Interagencial para América Latina y el Caribe. Washington D.C.: OPS, 2003.

13. Organización de las Naciones Unidas. Objetivos de Desarrollo del Milenio: La progresión hacia el derecho a la salud en América Latina y el Caribe [En línea]. 2009 [Consultado el 23 de noviembre de 2013]. Disponible en: http://www.unicef.org/lac/2008ODMSaludcompletoFinalsh.pdf

14. Organización Panamericana de la Salud / OMS. Clasificación estadística internacional de enfermedades y problemas relacionados con la salud. Décima revisión (CIE-10) [En línea]. Washington, D.C. 1995 [Consultado el 23 de noviembre de 2013]. Disponible en: http://ais.paho.org/classifications/Chapters/pdf/Volu me2.pdf

15. Safe Motherhood [En línea]. New York: Safe Motherhood Inter-Agency Group; 2002 [Consultado el 3 de Octubre de 2014]. [aprox. 2 pantallas]. Disponible en: http://www.safemotherhood.org/priorities/index.htm 1

16. Duarte T, De la Cruz M. La muerte materna: Sucesos y consecuencias para los huérfanos, las familias y las comunidades. Estudio de caso en Alta Verapaz [En línea]. 2010 [Consultado el 3 de Octubre de 2014]. Disponible en: http://mspas.gob.gt/decap/files/descargas/biblioteca Virtual/Documentos\%20de\%20salud/La\%20muerte\% 20materna.pdf\#

17. Gomes F, Nakano A, Almeida A, Matuo Y. Mortalidade materna na perspectiva do familiar. Rev. esc. enferm. 2006;40(1):50-56.

18. Rodríguez D, Verdu J. Consecuencias de una muerte materna en la familia. Aquichán. 2013;13(3):433-441.

19. Sáenz S. Consecuencias de la muerte materna: estudio de caso en Alta Verapaz. Informe final de investigación. 2009, Disponible en: http://www.academia.edu/6619496/Consecuencias_d e_la_muerte_materna_en_Guatemala

20. Herrera MC, Cruz JL, Robledo GP, Montoya G. La economía del grupo doméstico: determinante de muerte materna entre mujeres indígenas de Chiapas, México. Rev Panam Salud Pública. 2006;19(2):69-78.

21. Reyes S, Bobadilla J, Karchmer S, Martínez L. Efecto de la muerte materna sobre la dinámica familiar y la sobrevivencia infantil. Ginec. Obst. Mex. 1998; 66(1):428-433.

22. Zubrick SR, Mitrou F, Lawrence D, Silburn SR. Maternal death and the onward psychosocial circumstances of Australian Aboriginal children and young people. Psychol Med. 2011;41(9):1971-80

23. Ramírez MF, Rivera M, Durón R, Aguilar O, Fuentes M, Gómez C, Claudino C, Batres Y, Almendares J. Impacto de 
la mortalidad materna en la familia, especialmente en la niñez de Occidente de Honduras. Rev Med Hond. 2003; 71(1):114-122.

24. Portal RE. Consecuencias familiares y sociales de la muerte materna en la sub región de salud Cajamarca, Perú 2009; Disponible en: http://www.gycperu.com/descargas/5_setiembre/Mu erte\%20Materna\%20en\%20Cajamarca.pdf

25. Durán E, Valoyes E. Perfil de los niños, niñas y adolescentes sin cuidado parental en Colombia. Rev. latinoam. cienc. soc. niñez juv. 2009;7(2):761-783.

26. Case A, Ardington C. The impact of parental death on school outcomes: longitudinal evidence from South Africa. Demography (pre-2011) 2006;43(3):401-20.

27. Wang H, Ye F, Wang Y, Huntington D, China. Economic impact of maternal death on households in rural china: A prospective cohort study. PLoS One. 2013;8(10):1-14.

28. Ye F, Wang H, Huntington D, Zhou H, Li Y, You F, Li J, Cui W, Yao M, Wang Y, China MD. The immediate economic impact of maternal deaths on rural chinese households. PLoS One. 2012;7(6).

29. Gellego VL, Velez AG y Agudelo B. Panorama de la mortalidad materna. En: Modelos para el análisis de la mortalidad materna y perinatal. Medellín: Secretaría de Salud; 2005; 15-31.

30. Yamin A, Boulanger M, Falb L, Shuma J, Leaning J. Costs of inaction on maternal mortality: qualitative evidence of the impacts of maternal deaths on living children in Tanzania. PLoS One. 2013;8(8).

31. Sipos L, Solano C. El duelo en los niños. Experiencia clínica en un Centro de Salud Mental. Rev. Psiquiatría 2002;6(5).

32. Durán E, Valoyes E. Perfil de los niños, niñas y adolescentes sin cuidado parental en Colombia. Rev. latinoam. cienc. soc. niñez juv. 2009;7(2):761-783.

33. Chen SH, Chen Y, Liu J. The Impact of unexpected maternal death on education: First evidence from Three National Administrative Data Llinks. Am Econ Rev. 2009; 99(2):149-153

34. Pelloso S, Guarino M. A problemática familiar e a morte materna. Ciência, Cuidado e Saúde. 2006;5(1):19-25.

35. Pavard S, Gagnon A, Desjardins B, Heyer E. Muerte de la madre y supervivencia infantil: El caso de principios de Québec. J biosoc Sci. 2005;37:209-27.

36. Atrash HK. Parents' death and its implications for child survival. Rev Bras Crescimento Desenvolvimento Hum. 2011;21(3):769-770.

37. Nakiyingi J, Bracher M, Whitworth J, Ruberantwari A, Busingye J, Mbulaiteye S, Zaba B. Child survival in relation to mother's HIV infection and survival: evidence from a Ugandan cohort study. AIDS. 2003;17:18271834.

38. Clark SJ, Kahn K, Houle B, Arteche A, Collinson MA, Tollman SM, Stein A. Young children's probability of dying before and after their mother's death: A Rural South African Population-Based Surveillance Study. PLoS Medicine. 2013;10(3).

39. Mishra V, Arnold F, Otieno F, Cross A, Hong R. Education and nutritional status of orphans and children of HIVinfected parents in Kenya. AIDS Education and Prevention. 2007;19(5):383-95.
40. Katz J, West KP, Khatry SK, Christian P, LeClerq SC, Kimbrough E, Sherstha SR. Risk factors for early infant mortality in Sarlahi district, Nepal. World Health Organization. Bulletin of the World Health Organization. 2003;81(10):717-25.

41. Ronsmans C, Chowdhury ME, Dasgupta SK, Ahmed A, Koblinsky M. Effect of parent's death on child survival in rural Bangladesh: a cohort study. The Lancet. 2010; 375(9730):2024-31.

42. Becher H, Muller O, Jahn A, Gbangou A, Kynast G, Kouyaté B. Risk factors of infant and child mortality in rural Burkina Faso. World Health Organization. Bulletin of the World Health Organization. 2004;82(4):265-73.

43. Karam M, Bustamante P, Campuzano M, Camarena A. Aspectos sociales de la mortalidad materna. Estudio de caso en el Estado de México. Medicina Social. 2007;2(4): 205-211.

44. Donald N, Tsheko S, Mtero-MunyatiM. Segwabe P. Chibatamoto S. Mfecane B. Chandiwana N. Nkomo S. Tlou G. Chitiyo. Towards a definition of orphaned and vulnerable children. AIDS and Behavior. 2006; $10(6): 619-26$.

45. Yusuf HR, Atrash HK. Parents' death and survival of their children. The Lancet. 2010;375(9730):1944-6

46. Elegbeleye AO. Predictors of the mental health of orphans and vulnerable children in Nigeria. Ife Psychologia. 2013;21(2):170-180

47. Organización Mundial de la Salud. Reducción de la mortalidad materna. Declaración conjunta OMS/FNUAP/UNICEF/Banco Mundial [En línea] 1999. http://whqlibdoc.who.int/publications/1999/924356 1952_spa.pdf?ua=1

48. República de Colombia. Instituto Nacional de Salud. Grupo Funcional Maternidad Segura. Boletín mortalidad materna [En línea]. 2014 [Consultado el 3 de Octubre de 2014]. [aprox. 9 pantallas]. http://www.minsalud.gov.co/sites/rid/Lists/Bibliotec aDigital/RIDE/IA/INS/CES\%20MORTALIDAD\%20MAT ERNA\%20SEMANA\%2038.pdf

49. Fondo de las Naciones Unidas para la Infancia- UNICEF. Salud materna, [En línea]. 2014 [Consultado el 3 de Octubre de 2014]. [aprox. 2 pantallas]. Disponible en: http://www.unicef.com.co/situacion-de-lainfancia/salud-materna/

50. Ruiz-Matus C. Salud materna: necesidad de establecer alianzas. PerinatolReprodHum. 2005;19:1-3. 